\title{
Biodegradação dos herbicidas imazetapir e imazapique em solo rizosférico de seis espécies vegetais
}

\author{
Biodegradation of the herbicides imazethapyr and imazapic in rhizosphere \\ soil of six plant species
}

\author{
Kelen Müller Souto ${ }^{I}$ Rodrigo Josemar Seminoti Jacques ${ }^{\text {II }}$ Luis Antonio de Avila ${ }^{\text {II }}$ \\ Sérgio Luiz de Oliveira MachadoII Renato Zanella ${ }^{\text {II }}$ João Paulo RefattiII
}

\section{RESUMO}

A fitoestimulação é uma das técnicas de fitorremediação que pode ser utilizada para a redução da concentração dos herbicidas no solo. Para tanto é necessário a seleção de plantas que estimulem a atividade dos microrganismos degradadores da rizosfera. O objetivo deste trabalho foi avaliar a biodegradação de cinco doses do herbicida composto pela mistura formulada de imazetapir+imazapique $\left(75+25\right.$ g e.a. $\left.L^{-1}\right)$ em solo rizosférico proveniente de seis espécies vegetais com potencial para a fitoestimulação. A biodegradação do herbicida nas doses de 0, 250, 500, 1.000 e 4.000mL ha-1 no solo rizosférico de Canavalia ensiformis, Glycine max, Lolium multiflorum, Lotus corniculatus, Stizolobium aterrimum, Vicia sativa e no solo sem cultivo foi avaliada através da quantificação da produção de $\mathrm{C}-\mathrm{CO}_{2}$ do solo e da degradação do herbicida no solo por cromatografia líquida acoplada à espectrometria de massa. A produção de $\mathrm{C}-\mathrm{CO}_{2}$ do solo após a contaminação com a mistura de imazetapir+imazapique foi maior nos solos rizosféricos das seis espécies vegetais se comparado ao solo não cultivado. $O$ solo rizosférico de Stizolobium aterrimum apresentou a maior degradação do herbicida imazetapir nas maiores doses avaliadas, sendo uma espécie promissora para a fitoestimulação.

Palavras-chave: arroz irrigado, rizosfera, microrganismos, fitorremediação, fitoestimulação.

\section{ABSTRACT}

Phytostimulation is a technique of phytoremediation that can be used to reduce the concentration of herbicides in the soil. It is necessary to select plants that stimulate the activity of the rizosphere degrading microorganism. The aim of this study was to evaluate the activity of the biodegradation of the herbicide compound formulated mixture of imazethapyr + imazapic $\left(75+\right.$ and $\left.25 \mathrm{~g} \mathrm{~L}^{-1}\right)$ in rhizosphere soil from six plant species with potential for phytostimulation. Biodegradation of the herbicide in the rhizosphere soil of Canavalia ensiformis, Glycine max, Lolium multiflorum, Lotus corniculatus, Stizolobium aterrimum and Vicia sativa, and a control (soil without plant) was evaluated at doses of 0,250,500, 1.000 and 4.000mL 1 ha, by quantifying the production of $\mathrm{C}-\mathrm{CO}_{2}$ of the soil and the degradation of the herbicide from the soil by liquid chromatography coupled to mass spectrometry. The production of $\mathrm{C}-\mathrm{CO}_{2}$ of the soil after the contamination with the mixture imazethapyr + imazapic was higher in the rhizosphere soil of the six plant species when compared to soil not cultivated. The rhizospheric soil from Stizolobium aterrimum shouwed the highest degradation of the herbicide imazetapyr on the highest doses evaluated, being a promising specie for phytostimulation.

Key words: rice, rhizosphere, microorganisms, phytoremediation, phytostimulation.

\section{INTRODUÇÃO}

Em solos cultivados com determinadas espécies de plantas tem sido observada uma redução mais rápida dos contaminantes, em comparação aos solos sem cultivo ou aos solos cultivados com outras espécies (SANTOS et al., 2009). Esse efeito geralmente é atribuído ao maior número de microrganismos degradadores na rizosfera de algumas plantas, consequência das rizodeposições de nutrientes, tais como aminoácidos, polissacarídeos e outros compostos específicos (PIRES et al., 2005).

A ação desses organismos rizosféricos no aumento da taxa de degradação dos contaminantes

\footnotetext{
'Departamento de Defesa Fitossanitária, Universidade Federal de Santa Maria (UFSM), 97105-900, Santa Maria, RS, Brasil. E-mail: kelen ms@yahoo.com.br. Autor para correspondência.

IIUSM $\overline{\text { I }}$, Santa Maria, RS, Brasil.

IIIniversidade Federal de Pelotas (UFPel), Pelotas, RS, Brasil. 
orgânicos no solo é conhecida como fitoestimulação e constitui-se em um das principais técnicas de fitorremediação de herbicidas no solo (SANTOS et al, 2010). Vários trabalhos demonstraram a contribuição positiva da fitoestimulação para o aumento da degradação de herbicidas no solo, em especial da atrazina, do metalaclor, do trifloxisulfuron-sódio, do glifosato e do imazapir (PERKOVICH et al., 1996; SANTOS et al., 2010; SOUZA, et al., 1999), porém há escassez de informações em relação aos herbicidas imazetapir e imazapique, pertencentes ao grupo químico das imidazolinonas.

Esses herbicidas são usualmente utilizados em áreas de várzea de cultivo do arroz irrigado por inundação, manejadas sob o Sistema Clearfield ${ }^{\circledR}$. De acordo com STEELE et al. (2002), esse Sistema utiliza cultivares de arroz irrigado tolerantes aos herbicidas do grupo químico das imidazolinonas, o que possibilita o controle do arroz vermelho (Oryza sativa L.), principal planta daninha dessa cultura agrícola. Quando aplicados na lavoura, uma proporção significativa desses herbicidas atinge o solo, onde são passíveis de serem absorvidos pelas raízes das plantas e/ou sorvidos aos coloides do solo (KRAEMER et al., 2009).

Uma das características dos herbicidas imazetapir e imazapique é a sua longa persistência no solo (VILLA, et al., 2006), o que resulta na redução do número de aplicações de herbicidas para o controle das plantas daninhas. No entanto, tem-se observado a ocorrência de fitointoxicação em plantas sensíveis (carryover), cultivadas após a utilização desses herbicidas (VILLA et al., 2006; PINTO et al., 2010), cujo efeito residual varia de alguns meses até três anos ou mais, dependendo das características do ambiente em que se encontram (SENSEMAN, 2007). A elevada atividade da molécula no solo, a sua persistência por longos períodos e a extrema sensibilidade de algumas culturas a esses herbicidas impõem restrições ao uso de determinadas culturas em rotação/sucessão ao arroz irrigado manejado sob o Sistema Clearfield ${ }^{\circledR}$ (ROMAN et al., 2007). Além disso, quanto maior o tempo de permanência do herbicida no solo, maior é a possibilidade de contaminação de outros compartimentos ambientais, como as águas subsuperficiais, através da lixiviação do próprio herbicida e/ou de seus metabólitos (PIRES et al., 2003).

Uma alternativa para a redução da concentração dos herbicidas do grupo das imidazolinonas do solo é a fitorremediação. Como um dos principais mecanismos de dissipação destes herbicidas no ambiente é a degradação microbiana (FLINT \& WITT, 1997), torna-se fundamental a identificação de espécies de plantas que estimulem o crescimento de microrganismos degradadores na rizosfera, o que pode resultar no aumento das taxas de biodegradação do herbicida no solo. Em vista do exposto, esse trabalho teve por objetivo avaliar a biodegradação de cinco doses do herbicida composto pela mistura formulada de imazetapir+imazapique $(75+25$ g e.a. $\left.\mathrm{L}^{-1}\right)$ em solo rizosférico proveniente de seis espécies vegetais com potencial para a fitoestimulação.

\section{MATERIAL E MÉTODOS}

O experimento foi realizado em dois momentos, de acordo com a estação de cultivo das espécies testadas. O cultivo das espécies vegetais foi realizado em casa de vegetação, em vasos contendo $3 \mathrm{dm}^{3}$ de solo de várzea, livre de herbicidas. O solo utilizado pertence à unidade de mapeamento Vacacaí, coletado no horizonte A e classificado como Planossolo Háplico Eutrófico arênico (EMBRAPA, 2006), com classe textural franco siltosa e as seguintes características: $\mathrm{pH}_{(1: 1 \mathrm{H} 2 \mathrm{O})}=6,2$; argila $=$ $200 \mathrm{~g} \mathrm{~kg}^{-1}$, matéria orgânica (Walkley-Black) $=32 \mathrm{~g} \mathrm{~kg}^{-1}$, $\mathrm{P}_{(\text {Mehlich-1) }}=16,2 \mathrm{mg} \mathrm{dm}^{-3}, \mathrm{~K}_{(\text {Mehlich-1) }}=180 \mathrm{mg} \mathrm{dm}^{-3}$, $\mathrm{Ca}_{(\mathrm{KCl} 1 \mathrm{M})}=5,5 \mathrm{cmol}_{\mathrm{c}} \mathrm{dm}^{-3}, \mathrm{Mg}_{(\mathrm{KCl} \mathrm{lM})}=3,2 \mathrm{cmol}_{\mathrm{c}} \mathrm{dm}^{-3}$, $\mathrm{Al}_{(\mathrm{KCl} \mathrm{IM})}=0,0 \mathrm{cmol}_{\mathrm{c}} \mathrm{dm}^{-3}$ e índice $\mathrm{SMP}=6,6 . \mathrm{O}$ solo foi previamente corrigido, em relação à acidez e ao teor de nutrientes, de acordo com as recomendações para a cultura da soja. As espécies estivais Canavalia ensiformis (feijão-de-porco), Glycine max (soja) e Stizolobium aterrimum (mucuna-preta) foram cultivadas no período de março a maio de 2010 e as espécies hibernais Lolium multiflorum (azevém anual), Lotus corniculatus (cornichão) e Vicia sativa (ervilhaca) foram cultivadas no período compreendido entre os meses de junho a agosto do mesmo ano.

Cinquenta e cinco dias após a emergência, as plantas inteiras foram retiradas dos vasos e o solo aderido às raízes foi considerado como sendo de solo rizosférico (SANTOS et al., 2009). Este solo recebeu imediatamente a aplicação de diferentes concentrações dos herbicidas, conforme descrito abaixo. Simultaneamente, as amostras de solo mantidas sob as mesmas condições, mas sem cultivo (solo não rizosférico) foram utilizadas como controle.

A biodegradação dos herbicidas foi quantificada por meio de dois experimentos respirométricos, em condições de laboratório, de acordo com a estação de cultivo das plantas testadas, 
e pela quantificação cromatográfica da concentração dos herbicidas no solo (LC-MS-MS).

Experimento de verão - as amostras de solo rizosférico das espécies estivais foram peneiradas em malha de $2,0 \mathrm{~mm}$ de abertura, secas à sombra por 24 horas, pesadas e $100 \mathrm{~g}$ acondicionadas em frascos respirométricos de vidro de 1L. Logo após, uma solução de imazetapir+imazapique, na concentração de $75+25$ g e.a. $\mathrm{L}^{-1}$ do produto técnico foi adicionada em diferentes doses, utilizando-se pipetador de precisão. Foi feita a adição de água necessária para alcançar o mesmo teor de umidade em todas as amostras ( $80 \%$ da capacidade de campo do solo). $\mathrm{O}$ experimento foi conduzido no delineamento inteiramente casualizado, em esquema fatorial $4 \times 5$, contendo três repetições. Os tratamentos foram compostos pela combinação de amostras de solo rizosférico de três espécies vegetais: C. ensiformis, G. $\boldsymbol{m a x}$ e $\boldsymbol{S}$. aterrimum, mais a amostra de solo não cultivado; e de cinco doses da mistura formulada de imazetapir+imazapique: zero, 250, 500, 1.000, $4.000 \mathrm{~mL} \mathrm{ha}^{-1}$, totalizando 60 unidades experimentais.

Experimento de inverno - $\mathrm{O}$ segundo experimento respirométrico foi conduzido da mesma forma que o primeiro, porém com o solo rizosférico das espécies hibernais $\mathbf{L}$. multiflorum, L. corniculatus e $\boldsymbol{V}$. sativa, mais a amostra de solo não cultivado.

Os frascos respirométricos de vidro foram equipados com aparato de captura de $\mathrm{CO}_{2}$, composto por um copo plástico de $50 \mathrm{~mL}$ com $20 \mathrm{~mL}$ de $\mathrm{NaOH}$ 0,5M, fechados hermeticamente e incubados em triplicata, à temperatura ambiente no laboratório (20 a $25^{\circ} \mathrm{C}$ ). Durante um período de 63 dias, semanalmente os frascos foram abertos e a solução de $\mathrm{NaOH}$ recebia um $\mathrm{mL}$ de $\mathrm{BaCl}_{2} 1 \mathrm{M}$ e procedia-se à titulação com $\mathrm{HCl} 1 \mathrm{M}$, utilizando fenolftaleína como indicador. A solução de $\mathrm{HCl}$ foi padronizada com a solução de TRIS ou THAM (tris-hidroximetil amino metano) (Merck ${ }^{\mathrm{TM}}$ ), conforme TEDESCO et al. (1995). Dois frascos foram incubados sem solo, sendo estes utilizados como prova em branco. A produção de $\mathrm{C}-\mathrm{CO}_{2}$ foi quantificada através da fórmula de STOTZKY (1965).

Ao final do período de biodegradação dos herbicidas (63 dias), as amostras de solo foram acondicionadas em sacos de papel e encaminhadas ao Laboratório de Análise de Resíduos de Pesticidas (LARP-UFSM), onde foi realizada a detecção e a quantificação dos herbicidas imazetapir e imazapique ainda existentes nas amostras de solo rizosférico após o experimento respirométrico. Para isso utilizou- se a técnica de cromatografia líquida acoplada à espectrometria de massa (LC-MS-MS) descrita por ZANELLA et al. (2003), onde as amostras foram acidificadas e pré-concentradas em cartuchos contendo 500mg do adsorvente STRATA C18, sendo a diluição executada por duas vezes com $500 \mu \mathrm{L}$ de metanol. A detecção e a quantificação dos herbicidas imazetapir e imazapique foram realizadas utilizandose HPLC-UV, a $220 \mathrm{~nm}$, equipado com uma coluna Bondesil C18 $(250 \times 4,6 \mathrm{~mm}$ i.d; $5 \mu \mathrm{m})$, com fase móvel constituída de metanol e água $\left(60: 40 \mathrm{vv}^{-1}\right)$, ajustada a pH 4,0 com ácido fosfórico, com vazão de $0,8 \mathrm{~mL} \mathrm{~min}^{-1}$.

Os dados foram analisados quanto a homogeneidade da variância e normalidade, sendo submetidos aos procedimentos de análise da variância e regressão polinomial, através do programa computacional SAS, para a análise estatística, e SigmaPlot ${ }^{\circledR}$, para confecção das equações de regressão referentes à taxa de produção de $\mathrm{C}-\mathrm{CO}_{2}$.

\section{RESULTADOS E DISCUSSÃO}

Os 63 dias de duração do experimento respirométrico foram suficientes para uma adequada estimativa da quantidade de $\mathrm{C}-\mathrm{CO}_{2}$ produzido, uma vez que, ao final desse intervalo de tempo a produção de $\mathrm{C}-\mathrm{CO}_{2}$ apresentou tendência de estabilização, em todas as concentrações avaliadas da mistura herbicida (dados não apresentados).

Houve tendência de maior produção de $\mathrm{C}-\mathrm{CO}_{2}$ quanto maior a concentração do herbicida aplicada no solo rizosférico das espécies hibernais (Figura 1A) e estivais (Figura 1B). Porém, o comportamento polinomial quadrático das curvas mostra que menor produção de $\mathrm{C}-\mathrm{CO}_{2}$ ocorreu na dose de $4.000 \mathrm{~mL} \mathrm{ha}^{-1}$, que representa mais que o dobro da dose de campo normalmente recomendada para esse herbicida, que é de $1.500 \mathrm{~mL} \mathrm{ha}^{-1}$. Essa menor porcentagem de mineralização na maior dose aplicada pode ser devido ao efeito tóxico do herbicida sobre os microrganismos do solo rizosférico, comprometendo a sua atividade. ZHANG et al. (2010) relatou que o herbicida imazetapir aplicado em solo cultivado com soja não teve efeitos adversos sobre a biomassa microbiana do solo quando aplicado na dose de campo, porém teve efeito tóxico quando aplicado em taxas mais elevadas. Em trabalho realizado por PIRES et al. (2005), em solo rizosférico de mucuna-anã (Mucuna deeringiana), ocorreu queda na produção de $\mathrm{CO}_{2}$, explicada pelo provável efeito tóxico provocado pelas doses mais 


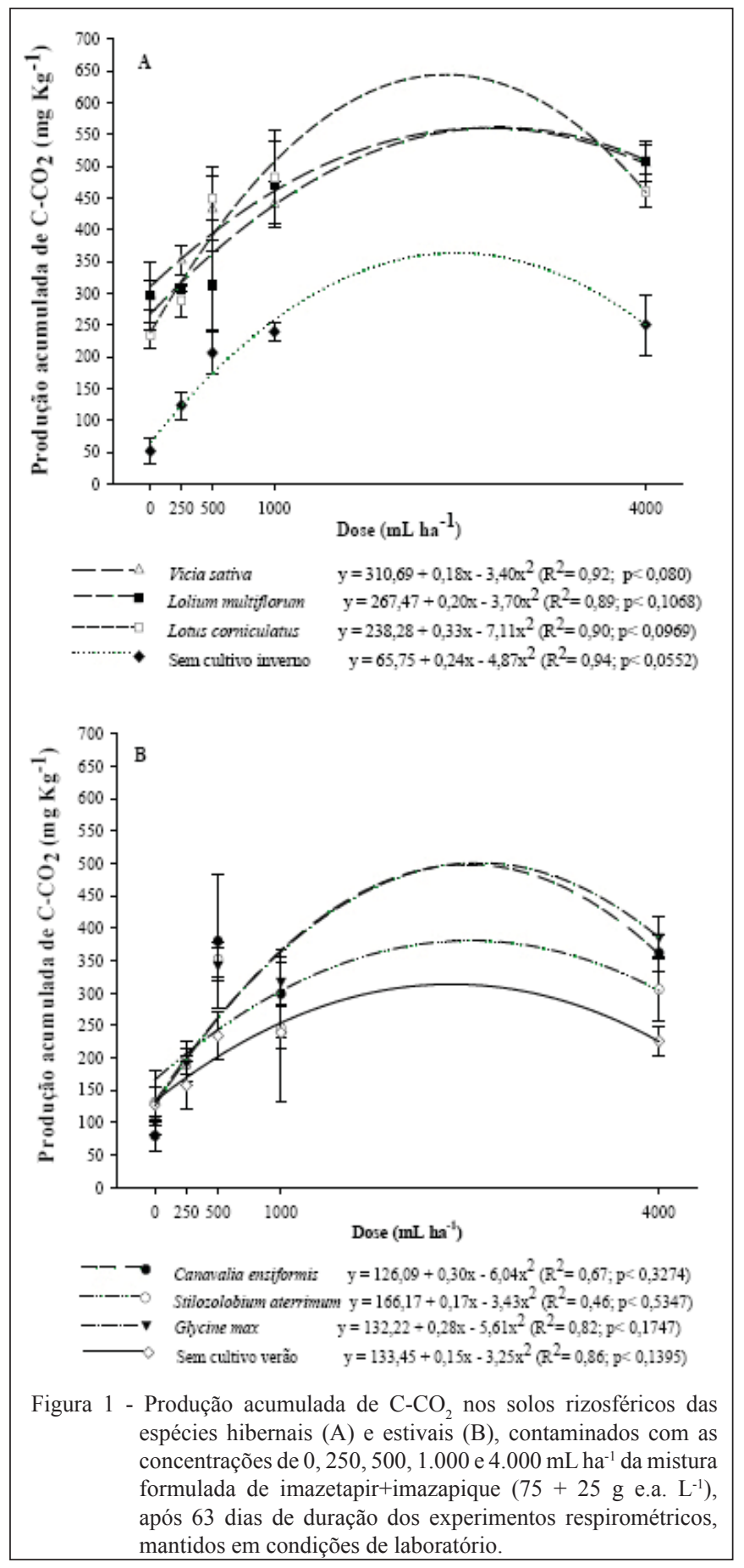

elevadas do herbicida tebutiuron sobre a microbiota. Os resultados do presente trabalho e dos autores acima citados remetem à possibilidade de que após sucessivas aplicações de herbicidas do grupo das imidazolinonas, pode ocorrer redução da degradação microbiana no ambiente, o que conduziria a maior persistência dos herbicidas no solo e o maior efeito fitotóxico na cultura em sucessão.
A produção acumulada de $\mathrm{C}-\mathrm{CO}_{2}$ foi maior no solo cultivado com as espécies hibernais L. corniculatus, L. multiflorum e V. sativa, comparado com o solo sem cultivo, independente da dose herbicida aplicada, sendo que essas não diferiram significativamente entre si, exceto na dose de $250 \mathrm{~mL} \mathrm{ha}^{-1}$, onde a espécie $\boldsymbol{V}$. sativa foi superior às demais (Figura 1A). Corroborando com 
resultados, PIRES et al. (2005) observaram que o solo rizosférico tratado com tebutiuron apresentou maior produção de $\mathrm{CO}_{2}$, se comparado ao solo não vegetado, sendo esse fato atribuído à fitoestimulação da microbiota associada à rizosfera. Não houve diferenças estatísticas na produção de $\mathrm{C}-\mathrm{CO}_{2}$ entre as três espécies estivais avaliadas. No entanto, o solo não cultivado novamente apresentou em todas as doses o menor acúmulo de $\mathrm{C}-\mathrm{CO}_{2}$ diferindo significativamente do solo rizosférico das espécies estivais nas doses de 500 e $4.000 \mathrm{~mL} \mathrm{ha}^{-1}$.

É provável que a maior atividade observada nos solos cultivados e contaminados com o herbicida, em comparação com o solo sem cultivo, nas duas estações, se deva à maior população de microrganismos, devido à habilidade que, provavelmente, essas plantas possuem em rizodepositar uma gama de moléculas de alto e baixo peso molecular, que resulta na maior proliferação de microrganismos dentro da raiz (endorrizosfera), sobre a raiz (rizoplano) e ao redor da raiz (ectorizosfera) (MARRIEL et al., 2005). Outra possibilidade é que a microbiota associada às raízes dessas plantas possam, além de tolerar os herbicidas imazetapir e imazapique, utilizá-los como substrato para seu metabolismo, como ocorre com os microrganismos existentes rizosfera da espécie Kochia scoparia, fitorremediadora de atrazine (PERKOVICH et al., 1996), e com Helianthus annus, potencialmente fitorremediadora de sulfentrazone (BELO et al., 2011).

Comparando-se as espécies estivais e hibernais observa-se que, na média das doses avaliadas, houve maior produção de $\mathrm{C}-\mathrm{CO}_{2}$ no solo rizosférico das espécies estivais, sem haver diferenças estatísticas entre os dois grupos de plantas. Cabe ressaltar, no entanto, que a produção de $\mathrm{C}-\mathrm{CO}_{2}$ quantificada nesses experimentos respirométricos, pode ser proveniente não só da mineralização das moléculas da mistura formulada de imazetapir+imazapique $\left(75+2 \mathrm{~g}\right.$ e.a. $\left.\mathrm{L}^{-1}\right)$ aplicada no solo, mas também da mineralização da matéria orgânica presente no solo, bem como dos próprios compostos liberados pelas raízes das plantas (rizodepozição) ou dos compostos adjuvantes existentes na formulação da mistura herbicida utilizada. Essas diferentes fontes de $\mathrm{C}-\mathrm{CO}_{2}$ acima mencionadas podem dificultar a quantificação da correta fração do herbicida que é degradada pela microbiota do solo.

Assim, para evitar interpretações errôneas, a detecção e a quantificação dos herbicidas imazetapir e imazapique foram realizadas nos solos rizosféricos após os experimentos respirométricos, utilizando-se a técnica de cromatografia acoplada a espectrometria de massas (LC-MS-MS). Os resultados mostraram uma elevada degradação dos herbicidas adicionados ao solo, em média de $93 \%$, independente da espécie vegetal e do cultivo ou não do solo (Figura 2). Porém nas maiores doses testadas houve diferenças estatísticas na degradação do imazetapir pelas espécies estivais. Na dose de $1.000 \mathrm{~mL} \mathrm{ha}^{-1}$, os solos rizosféricos das espécies $\boldsymbol{G}$. $\boldsymbol{m a x}$ e $\boldsymbol{S}$. aterrimum proporcionaram maiores degradações e na dose de $4.000 \mathrm{~mL} \mathrm{ha}^{-1}$ o solo rizosférico de $\boldsymbol{S}$. aterrimum apresentou a menor concentração do imazetapir, diferindo significativamente das demais espécies e do solo não cultivado. Esse comportamento indica que nas doses menores de 250 e $500 \mathrm{~mL} \mathrm{ha}^{-1}$ os microrganismos do solo rizosférico de todas as espécies avaliadas foram capazes de degradar, em média, $94 \%$ do herbicida adicionado ao solo, porém nas doses mais elevadas a atividade degradadora do solo rizosférico de algumas espécies se destacam, em especial da $\boldsymbol{S}$. aterrimum (Figura 2B). SANTOS et al. (2010) observaram que o solo contaminado com trifloxisulfuron-sódio e posteriormente cultivado com S. aterrimum apresentou maior atividade microbiana, que foi consequência da maior abundância de rizodeposições provenientes do desenvolvimento da espécie vegetal e da maior biomassa de microrganismos na rizosfera.

A elevada porcentagem de degradação dos herbicidas no solo observada no presente trabalho deve-se também às adequadas condições de umidade, temperatura e disponibilidade de nutrientes inorgânicos em que o experimento foi conduzido, propiciando alta atividade dos microrganismos rizosféricos. Condições estas que dificilmente ocorrem em condições de campo, o que pode resultar em menores porcentagens de biodegradação, se comparadas às condições do laboratório.

\section{CONCLUSÃO}

A produção de $\mathrm{C}-\mathrm{CO}_{2}$ do solo após a contaminação com a mistura formulada de imazetapir+imazapique é maior nos solos rizosféricos das seis espécies vegetais se comparado ao solo não cultivado.

O solo rizosférico de Stizolobium aterrimum apresenta a maior degradação do herbicida imazetapir nas maiores doses avaliadas, sendo uma espécie promissora para a fitoestimulação. 


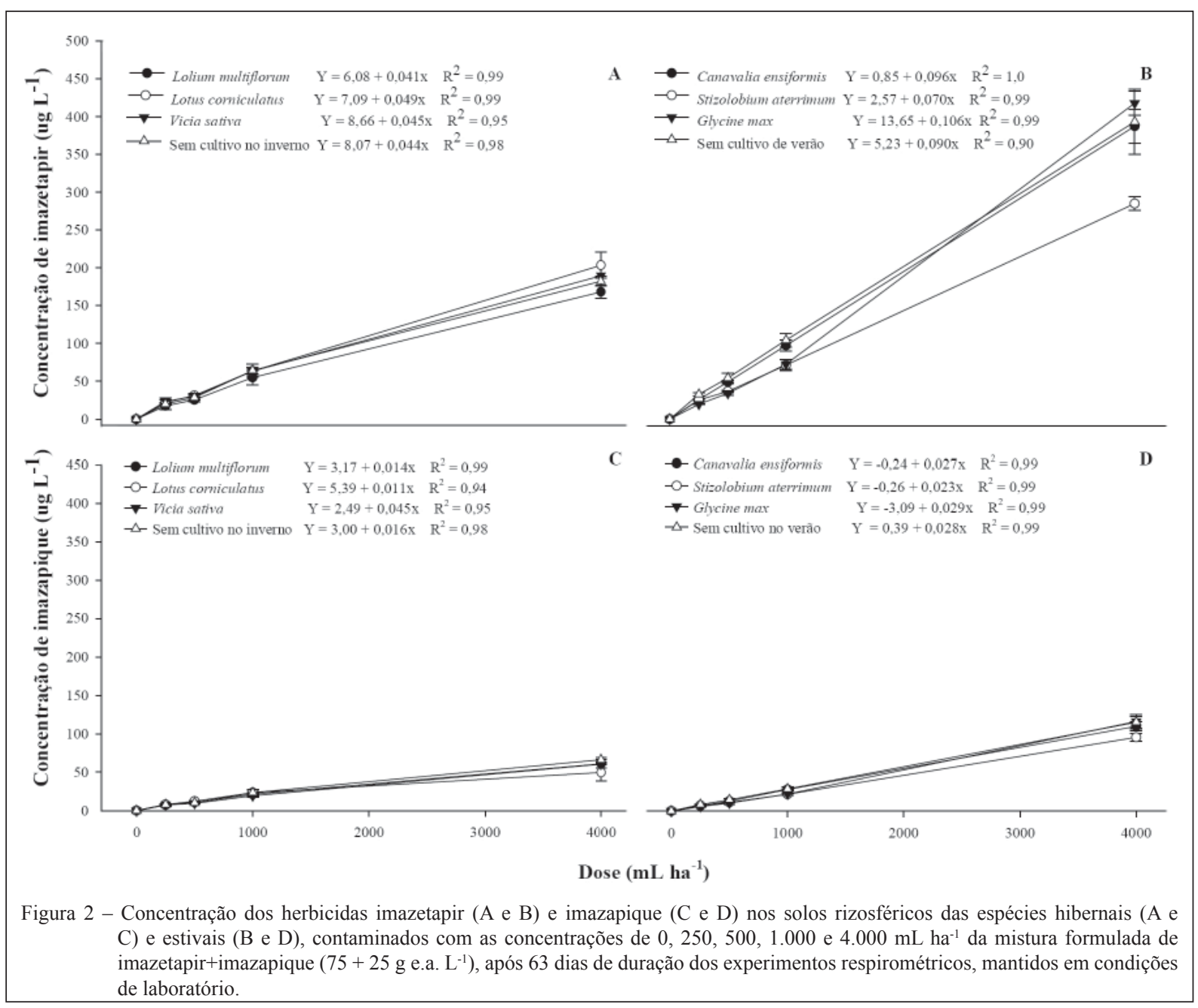

\section{REFERÊNCIAS}

BELO, A.F et al. Potencial de espécies vegetais na remediação de solo contaminado com sulfentrazone. Planta Daninha. y. 29, n. 4, p. 821-828, 2011. Disponível em: <http://www.scielo. br/scielo.php?pid=S0100-83582011.0004.00012\&script $=$ sci arttext>. Acesso em: 10 nov. 2011. doi: 10.1590/S010083582011.0004 .00012 .

EMBRAPA. Sistema Brasileiro de Classificação de Solo. $2^{\mathrm{a}}$ ed., Rio de Janeiro: EMBRAPA, 306 p. 2006.

FLINT, J. F.; WITT, W. W. Microbial degradation of imazaquin and imazethapyr. Weed Science, v. 45, p. 586-591, 1997. Disponível em: <http://www.jstor.org/stable/4045996>. Acesso em: 22 nov. 2010 .

KRAEMER, A.F. et al. Persistência dos herbicidas imazethapyr e imazapic em solo de várzea sob diferentes sistemas de manejo. Planta Daninha, v. 27, n. 3, p. 581-588. 2009. Disponível em: $<\mathrm{http}$ ://www.scielo.br/scielo.php?pid=S0100$83582009000300020 \&$ script $=$ sci_arttext $>$. Acesso em: 04 jan. 2010. doi: 10.1590/S0100-83582009000300020.

MARRIEL, I. E. et al. Aplicação da Técnica Eletroforese em Gel de Gradiente Desnaturante (DGGE) na Caracterização de Microrganismos Dominantes na Rizosfera de Plantas Cultivadas em Solo Ácido. Minas Gerais: Embrapa Milho e Sorgo (Circular Técnica, 72), 8p, 2005. Disponível em: $<$ http://www. cnpms.embrapa.br/publicacoes/publica/2005/circular/Circ_72. pdf $>$. Acesso em: 25 jan. 2010.

PERKOVICH, B. S. et al. Enhanced mineralization of [14C] atrazine in K. scoparia rhizosferic soil from a pesticide contaminated site. Pesticide Science, v. 46, p. 391-396, 1996. Disponível em:<http://onlinelibrary.wiley.com/doi/10.1002/(SICI)10969063(199604)46:4\%3C391::AID-PS374\%3E3.0.CO;2-L/ pdf>. Acesso em 14 abr. 2010. doi: 10.1002/(SICI)10969063(199604)46:4<391::AID-PS374>3.0.CO;2-L.

PINTO, J. J. O. et al. Atividade residual de imazethapyr + imazapic para sorgo granífero (Sorghum bicolor) semeado em rotação com o arroz irrigado. Planta Daninha,Viçosa - MG, v. 27, n. especial, p. 1015 - 1024, 2009. Disponível em: <http://www.scielo.br/pdf/ pd/v27nspe/v27nspea15.pdf $>$. Acesso em: 10 abr. 2009. doi: 10.1590/S0100-83582009000500015.

PIRES, F. R. et al. Fitorremediação de solos contaminados com herbicidas. Planta Daninha, v. 21, n. 2, p.335-341, 2003. Disponível em: <http://www.scielo.br/scielo.php?pid=S0100$83582003000200020 \&$ script $=$ sci arttext $>$. Acesso em: 08 out. 2009. doi: $10.1590 / \mathrm{S} 0100-83582003000200020$. 
PIRES, F. R. et al. Inferências sobre atividade rizosférica de espécies com potencial para fitorremediação do herbicida tebuthiuron. Revista Brasileira de Ciência do Solo, v. 29, n. 4, p. 627-634, 2005. Disponível em: <http://www.scielo.br/pdf/rbcs/ v29n4/26111.pdf>. Acesso em: 08 out. 2009. doi: 10.1590/S0100068320050004.00015 .

ROMAN, E. S. et al. Como funcionam os herbicidas: da biologia á aplicação. Passo Fundo: Berthier, 2007. 160p.

SANTOS, E. A. Atividade rizosférica de solo tratado com herbicida durante processo de remediação por Stizolobium aterrimum. Pesquisa Agropecuária tropical, v. 40, n. 1, p. 1-7, 2010. Disponível em: <http://www.revistas.ufg.br/index.php/pat/ article/view/4670>. Acesso em: 06 set. 2010.

SANTOS, J. B. et al. Biodegradation of glyphosate in rhizospheric soil cultivated with Glycine max, Canavalia ensiformis e Stizolobium aterrimum. Planta Daninha, v. 27, n. 4, p. 781-787, 2009. Disponível em: <http://www.scielo. br/scielo.php?pid $=$ S0100-835820090004.00016\&script $=$ sci arttext>. Acesso em: 04 set. 2010. doi: 10.1590/S0100835820090004.00016 .

SENSEMAN, S. A. (Ed.). Herbicide handbook. 9 ed. Lawrence: Weed Science Society of America, 2007. 458p.

SOUZA, A. P. et al. Respiração microbiana do solo sob doses de glyphosate e de imazapyr. Planta Daninha, v. 17, n. 3, p. 387-398, 1999. Disponível em: <http://www.scielo.br/pdf/pd/ v17n3/07.pdf>. Acesso em: 06 set. 2010. doi: 10.1590/S010083581999000300007
STEELE, G. L. et al. Control of red rice (Oryza sativa) in imidazolinone-tolerant rice (Oryza sativa). Weed Technology, v. 16, n. 3, p. 627-630, 2002. Disponível em: <http://www.jstor. org $/$ discover $/ 10.2307 / 3989532$ ?uid=37656\&uid=3737664\&uid=2 $129 \&$ uid $=5909624 \&$ uid $=2$ \&uid $=70 \&$ uid $=3 \&$ uid $=67 \&$ uid $=37655$ \&uid $=62 \& \operatorname{sid}=21101099053583>$. Acesso em: 27 jan. 2011. doi: 10.1614/0890-037X(2002)016[0627:CORROS]2.0.CO;2).

STOTZKY, G. Microbial Respiration. In: BLACK, C. A. (ed.). Methods in Soil Analisys. Madison: ASSA, p. 1550-1572, 1965.

TEDESCO, M. J. et al. Análises de solo, plantas e outros materiais. 2 ed. Porto Alegre: Departamento de Solos da UFRGS, 1995. $174 \mathrm{p}$.

VILLA, S. C. C. et al. Arroz tolerante a imidazolinonas: controle do arroz-vermelho, fluxo gênico e efeito residual do herbicida em culturas sucessoras não-tolerantes. Planta Daninha, v.24, n.4, p. 761-768, 2006. Disponível em: <http://www.scielo.br/pdf/pd/ v24n4/a17v24n4.pdf>. Acesso em: 15 out. 2010. doi: 10.1590/ S0100-835820060004.00017

ZANELLA, R. et al. Development and validation of a highperformance liquid chromatographic procedure for the determination of herbicide residues in surface and agriculture waters. Journal of Separation Science, v.26, p.935-938, 2003. $<$ http://onlinelibrary.wiley.com/doi/10.1002/jssc.200301309/pdf $>$. Acesso em: 14 dez. 2010. doi: 10.1002/jssc.200301309

ZHANG, C. et al. The effect of imazethapyr on soil microbes in soybean fields in northeast China. Chemistry and Ecology, v. 26, n. 3, p. 173-182, 2010. Disponível em: <http://www.tandfonline. com/doi/abs/10.1080/02757541003785817\#preview>. Acesso em 15 de fev. 2012. doi: 10.1080/02757541003785817. 